\title{
An ICT Based Solution for Virtual Garment Fitting for Online Market Place
}

\author{
Hashini Gunatilake \\ University of Colombo School of Computing, Colombo 07, 00700, Sri Lanka \\ E-mail: hashini17s1@gmail.com
}

Dulaji Hidellaarachchi, Sandra Perera, Damitha Sandaruwan and Maheshya Weerasinghe

University of Colombo School of Computing, Colombo 07, 00700, Sri Lanka

E-mail: dulajidinupama@gmail.com, sandraperera1993@gmail.com,dsr@ucsc.cmb.ac.lk, amw@ucsc.cmb.ac.lk

Received: 16 August 2017; Accepted: 07 November 2017; Published: 08 February 2018

\begin{abstract}
In this paper, we describe various technologies that are being used in virtual garment fitting and simulation. There, we have focused on the usage of anthropometry in the clothing industry and avatar generation of virtual garment fitting. Most commonly used technologies for avatar generation in virtual environment have been discussed in this paper such as generic body model method and laser scanning technologies. Moreover, this paper includes the usage of real-time tracking technologies used in virtual garment fitting like markers and depth cameras. Apart from these, virtual clothing methods such as geometrical, physical and hybrid-based models were also discussed in this paper. As ease allowance has a major impact on virtual cloth fitting, it is also considered in this paper relating to similar research studies. As the final stage, our proposed design has been explained including the steps of the experiment that has been conducted to generate a twodimensional model of the garment item. Within this paper, all the above-mentioned areas were described thoroughly while stating the existing gap of the virtual garment fitting in online marketplaces and our proposed solution to bridge that gap.
\end{abstract}

Index Terms - Anthropometry, virtual garment fitting, avatar generation, generic body model, ease allowance.

\section{INTRODUCTION}

Nowadays people purchase garments online due to their busy lifestyles. It is visible that these online purchases have a considerable return rate. The main reason behind this situation is the size mismatch. There are many accurate computationally intensive $3 \mathrm{D}$ solutions to check the fitness of a garment with the human body. The problem associated with these $3 \mathrm{D}$ solutions is that we cannot use these to obtain a real-time result in the online marketplace since these require high human intervention and 3D mesh models of users and apparels. Further, there will be a large number of online users who will be accessing the system concurrently to check their fitness for a dress. Even though the available 3D solutions will provide an accurate solution, the time consumed by 3D solutions to deliver the solution will be very high. So, it can be seen that an online marketplace will require a quick solution and the available 3D solutions will not be appropriate to fulfill this requirement. Furthermore, according to a study done by D. Kim and K. LaBat (2013), considering the consumer experience in using 3D virtual garment simulation technology [1], most of the participants thought 3D virtual garment simulation would be a good starting point for judging fit, but there were some problems that the participants have experienced when using these technologies. The researchers have realized that there were privacy issues associated with the end users while using these $3 \mathrm{D}$ solutions since these technologies require the 3D body model of the user. The discomfort of viewing one's own body scan was a major issue while using these 3D virtual simulation technologies. Other than that, the lack of the availability of technology was also an issue. Therefore there is a need for a lightweight solution which will support the decision making of the end user in order to select the properly fitted dress by considering human body properties and garment properties.

\section{RELATED WORKS}

\section{A. Usage of Anthropometry in Clothing Industry}

According to Pheasant and Haslegrave (2006), anthropometry is a branch of human science which deals with the measurements of the human body in terms of size, shape, mobility, flexibility and working capacity [2]. It is observable that human body dimensions and proportions vary from one to another which triggers the necessity of understanding and analyzing the variances of the human body dimensions as well as the relationship among those. Pheasant and Haslegrave (2006) have presented correct standing and sitting postures in which the extraction of human body measurements could be obtained more accurately [2].

However, the inception of massive cloth production for 
the general public has triggered standardization of clothing sizes according to a clothing pattern grading system that was introduced by experts in the fashion industry. According to Schofield and LaBat (2005), aforementioned pattern grading method presents a standard way of making adjustments to cloth design patterns with reference to a predefined set of human body sizes [3]. This pattern grading system has a strong correlation with anthropometry. According to Huang (2012), the apparel industry uses ASTM D5219-09 and ISO 8559:1989 standards for taking anthropometric measurements and locating anatomical landmarks on human bodies [4]. These standards are used to locate body features on the parametric human models for which $3 \mathrm{D}$ garment should align with.

According to Chen (2008), there are eight body measurements required to be obtained for a normal trouser [5], and these parameters can be classified into two classes as follows: (1) Vertical type. Waist to hip, out-leg, curved front body rise. (2) Girth type. Thigh girth, waist girth, half back waist, hip size, half back hip.

This anthropometric system has contributed to the online garment purchasing process through the provision of a set of measurements representing different garment sizes where the shoppers are able to compare their own measurements against these size charts. The drawbacks of this comparison have influenced the development of virtual garment fitting.

\section{B. AVATAR GENERATION FOR VIRTUAL GARMENT FITTING}

Generation of avatars represents human body in various virtual fitting applications where human body measurements are considered as one of the main inputs which can be entered manually to generate an avatar. This is commonly used when using web-based applications, and apart from the human body measurements, the human body can also be extracted from numerous measuring and capturing technologies such as laser scanning, multi-camera capturing, motion detection and etc.

\section{- Use of Generic Body Models}

One popular method of representing virtual human body construction and animation is using generic models [6]. There, the creation of face, body skeleton, and body surface have been considered using the generic models. This avatar generation approach starts from default virtual human templates that include shape and animation structures. As one way, photographs have been used as inputs, where front and side views of the face and front, side and back views of the body were taken. This method is a feature-based modeling of a human body which can be animated. Following several steps, the generic model is animated and perform the animation with skin deformation. Then a generic body is gained with seamless surface and real-time skin deformation capability whenever skeleton joints are animated with given animation parameters.

In another way, certain measurements of the human body have been used to adjust the generic models to generate the avatar. There, they have taken eight measurements of length that have to be entered by the user. Based on these eight measurements particular segments of the avatar are adapted and others are done using interpolations.

Moreover, by creating generic human body model for standard five types of sizes (Extra Small, Small, Medium, Large, Extra-large) for each gender, and then taking the key body measurements the avatar generation process is carried out [7].

\section{- Use of Laser Scanning}

The creation of three-dimensional avatar can also be done using laser scanning [8]. Using 3D laser scanner, customer's body surface will be scanned within a few seconds and produces a three-dimensional point cloud. Then a mesh is generated out of these scanned point clouds and textures and acts as the basis for the virtual avatar.

By applying skin deformation methods to this created basis of a virtual avatar, the finalized avatar is capable of generating simple animations as well.

\section{- Use of Optical Tracking}

Use of optical tracking with commodity cameras is suggested in order to scan a person and then the scanned body mesh will be used as an avatar later [10]. To capture all sides of a person's body measurements, multi-camera setup with ten cameras will be used and compared to other methods, this is used because of its fast processing. With the pose estimation algorithms [11] the images are converted to a depth map and by using deformations and based on the images, the mesh is matched in order to resemble the proportions of the captured person [10].

A more natural look of avatar generation can be achieved using face detection algorithm on a photo of a user. Further adjustments such as skin color are done according to the face [9].

Though there are different ways of generating an avatar, most of these are unable to use in real-time applications as it requires huge computational power. As a result, there is a need for an avatar generation method which requires low computational power that can be used in online marketplace.

\section{Real-Time Tracking Technologies for Virtual Garment Fitting}

These technologies can be categorized into two parts as marker-based technologies and marker-less technologies. The marker-based approach uses different techniques. 'Fiducial markers' and optical markers are such techniques that come under this approach. Depth camera technique is known as a sophisticated marker-less technology which enables motion capturing and full body depth detection.

\section{- Markers}

The marker-based approach can be used as one of the methods to track shape of the body by placing these markers on predefined positions of the surface of the 
body. These are known as 'fiducial markers' since placement of markers is done in accordance with the comparison of placements of other markers.

In a study that presented an Augmented Reality application called ARDressCode has applied the concept of markers for their study [12]. Three cameras have been set-up to capture shopper's body and with the use of Augmented Reality Toolkit, the images captured by the camera are analyzed in order to place a set of predefined markers. As the final step, 3D model of the garment is positioned on body considering markers. In another study done in 2008 [13], researchers have applied markers on joints of a user using an algorithm that detects accurate positions of the body. Finally, a 2D garment is positioned on the body of the user based on markers.

With the introduction of marker-less tracking system, it was identified that marker-based approach is an errorprone method as the real-time positioning of markers were less accurate.

\section{- Depth Cameras}

In a study done in Japan, 3D body shapes of participants were obtained using depth cameras in order to overlay clothing images that are with similar body shape [14]. The subjects are captured with depth cameras, resulting single-shot depth images. This single-shot depth image is used to obtain 3D body model of subject with the application of depth data. Once single-shot depth image is rendered, shape of the body model is estimated using depth data. Finally, the most suitable clothing image with similar shape is selected from clothing database.

This method was able to produce considerably accurate garment fitting for the users, but with the high cost of large databases and equipment, the use of depth cameras is inappropriate for an online marketplace.

\section{Virtual Cloth Generation and Simulation}

Generation and simulation of virtual cloth can be done in various ways such as creating a 3D mesh to represent cloth, scanning an existing cloth using laser scanning techniques or using 2D planes with considering the texture of the fabric. From a research done at Graz University of Technology [10], they have generated the garment items virtually based on a multi-camera setup. With the assistance of this setup, a person will be captured who is wearing the actual cloth and the cloth should be separated from the body which can be done both manually or using 'chroma' key technique. In another method which is used for cloth generation [6][15], described in two research papers which were published in 2001 and 2003 respectively, first the outline of the cloth is drawn and placed on the body. After completing series of steps finally, the garment is adapted automatically according to the shape of the model. With a different technique that provides a realistic visualization of clothes is presented in a research [8], it has been mentioned that the finished results of cloth generation and simulation should give the real feeling of material properties to the users. In this method, clothes are created using 2D CAD geometry models and these models are typically utilized in the fashion industry.

Another research which was conducted in University of Geneva [7], they have used 3D garment simulator namely MIRACloth that was developed in University of Geneva. In this research, they have conducted the cloth simulation in two stages, garment assembly stage and garment animation stage.

\section{E. Virtual Clothing in Computer Graphics}

Virtual clothing supports the reproduction of physical behaviors and the visual features of textile objects in computer-simulated virtual reality [16]. Virtual clothing can be classified into three main categories such as geometrical based, physical-based and hybrid virtual clothing methods. The distinction among these categories is identified based on the core technique used for formulating shape of the cloth or for driving its deformation.

\section{- Geometrical Based Virtual Clothing Methods}

These techniques mainly concentrate on the properties related to appearance particularly folds and wrinkles which can be represented by geometrical equations. Physical properties of cloth will not be examined through these methods. Geometrical based virtual clothing methods require a substantial amount of user intervention which can be considered as one of its limitations.

The roots of geometrical virtual clothing methods spread back into 1986. Weil (1986) was able to introduce a way of modeling for three dimensional (3D) hanging cloth material [17]. Catenary curves between hanging points of cloth were used to induce shape of the cloth. This method can be used only to generate the hanging cloth and it cannot be used to generate a more complex cloth shape. T. Agui and Nakajima (1990) presented a way of modeling a sleeve on a bent arm [18]. According to their observation, the consequence of differences in curvature between inner and outer part of the sleeve has caused a formation of folds. This method focuses mainly the simulation of a bent sleeve. Hinds and McCartney (1990) aimed at automation of manufacturing garments [19]. In this method, upper body of a mannequin was digitized to obtain the shape of human body and numerous three dimensional (3D) panels were used to represent a garment. Afterwards, Hinds et al. (1991) proposed a way of translating three dimensional(3D) panels into two dimensional (2D) patterns using the method of Calladine (1986) [20] [21]. Miller et al. (1991) proposed a new approach to the issue of producing a simple topologically-closed geometric model from a point-sampled volume dataset [22]. A simple geometry as an initial topologically closed object such as a sphere or a cube was proposed through their research. Then the process of expanding this simple object in order to fit the object within a volume has been conducted. An extension of this method into cloth modeling was proposed by Thomas Stumpp (2008) [23]. Linear time complexity was reached considering a number of mesh vertices through their method. The physical property of cloth can be 
affected by the topology of cloth mesh as the physical property of the cloth is correlated to the size of clusters. Decaudin et al. (2006) introduced a method to create visually realistic clothes by wrapping developable surfaces around the character body in a natural manner [24]. The sewing patterns from 3D cloth model were provided by flattening the developable surfaces. A three dimensional (3D) surfaces were generated around character body using a sketch based interface and manually added the seam lines on these surfaces directly. A feature-based method for the construction of 3D cloth from 2D sketches was proposed by Wang et al. (2003) by considering predefined human body features as the base [25]. Tailoring rules in the fashion industry were used to define features of the human body based on the profiles of different body parts. 3D cloth templates are preconstructed considering each type of cloth. This method can generate only simple cloth meshes which needed to be processed further to complete a detailed cloth. An interactive way for putting and manipulating clothes on a 3D model was introduced by Igarashi \& Hughes (2002) [26]. 2D patterns are used to generate $3 \mathrm{D}$ cloth in this method and this can be used to model simple style cloth without folding and also because of the computational cost cloth-cloth collision is disregarded while calculating surface constraints of 3D cloth mesh. An approach for designing 3D cloth directly on a 3D mannequin model was introduced by Wang et al. (2009) considering constrained contour curves and style curves [27]. To define the general shape of clothes, contour curves such as silhouette curves and cross-section curves were used whereas to generate detailed 2D cloth patterns on cloth surfaces style curves such as seam lines, notch lines, and dart lines were used. This approach can be considered as an intuitive and appropriate way of designing complex cloth on a 3D mannequin. Further knowledge in fashion design and patternmaking is required to edit contour curves and style curves. Flexible shape control method was proposed Meng et al. (2012) for resizing 3D garments automatically while preserving the shape of user-defined features on clothes [28]. Any kind of cloth modeling techniques can be used to generate 3D clothes are modeled on a reference human body. Safeguarding of cloth features can be highly influenced by inconsistent user inputs since feature curves are defined by users based on each cloth modeled for target human bodies. An automatic way of cloth transferring between characters with different body shapes was introduced by Brouet et al. (2012) [29]. Vertices are adjusted on 3D cloth in order to fit a cloth onto a different character and pattern extraction will be happened after fitting $3 \mathrm{D}$ cloth to a new character through this method. Sketch-based interface for modeling 3D cloth on virtual characters was proposed by Turquin et al. (2007a) to model cloth on a 3D character based on stocks drawn by users [30]. This method can only be used to model simple style single layer clothes since information from user input stroke cannot be used to define complex cloth structure. An interactive tool was introduced by Umetani et al. (2011) for cloth design that enables bidirectional editing between $2 \mathrm{D}$ patterns and $3 \mathrm{D}$ cloth [31]. The real-time physics-based simulation method is used to simultaneously update the topology of $2 \mathrm{D}$ cloth pattern and its corresponding $3 \mathrm{D}$ cloth piece by user input, in order to maintain synchronization between $2 \mathrm{D}$ and 3D. The method proposed through this method cannot be used for simulation of cloth dynamic behavior subject to the motion of 3D character as synchronization of $2 \mathrm{D}$ pattern with a $3 \mathrm{D}$ cloth on a static $3 \mathrm{D}$ mannequin cannot be performed through this method.

\section{- Physical Based Virtual Clothing Methods}

Triangular or rectangular grids with points of fixed mass at intersections are used to represent cloth models in this physical based virtual clothing methods. Two types of models such as energy-based method (Terzopoulos et al. 1987) and force-based method (Volino \& MagnenatThalmann 2005) can be recognized in physical-based virtual clothing methods [32] [33]. The total energy of cloth is calculated using some equations in the energybased model. The shape of cloth is derived using these equations by moving points to achieve minimum energy state. In force-based models, forces among each point are represented as differential equations. The positions of points at each time step are obtained by solving these differential equations using numerical integration. A method for the construction of shape of a cloth object has been proposed by Terzopoulos et al. (1987) [32]. Shape and motion of deformable materials have been described using elasticity theory in this approach. Simulation of the dynamic behavior of objects can be implemented by fetching physical properties such as mass and damping into physical simulation. General mechanical model for cloth simulation was introduced by Volino and Magnenat-Thalmann (2005) for cloth simulation [33]. Instead of mass-spring system, an accurate particle system for dynamic simulation is presented through this model. Three 2D coordinates have designed considering three mechanical properties such as weft, warp elongation and shear and these were used to describe a triangle face of cloth mesh. A simulation model for large deformations of textile was proposed by Volino et al. (2009) [36]. Simulation process has become simple through this model and it enables the creation of nonlinear tensile behavior of textile with accuracy and robustness. An approach to simulate inextensible cloth in a collision-free condition subjected to a conservative force such as gravity was proposed by Chen and Tang (2010) [37]. Stretch resistance and compression resistance of a cloth is greater than its bending resistance according to this paper. Transformation of deformation process of an initial developable mesh surface to a final mesh surface through physical based simulation process has been proposed via this method.

In general, physical-based virtual clothing methods are used to produce behavior of a flexible object that resembles cloth. The rate of resemblance will vary based on used physical based virtual clothing technique.

\section{- Hybrid Virtual Clothing Methods}

Hybrid virtual clothing methods have been developed 
by combining both geometrical based virtual clothing methods and physical based virtual clothing methods to compensate deficiencies in those methods to deliver a proper solution. Rudomin (1990) proposed a method to diminish the computational complexity of physical based virtual clothing methods [38]. He presented a mechanism which can be used to lower computational time of physical based virtual clothing method which was introduced by Terzopoulos et al. (1987) [32]. After a while a series of hybrid virtual clothing methods were proposed by Kunii and Gotoda (1990) [39]; Tsopelas (1991) [40]. Geometrically modeled fine wrinkle details were mapped onto a physically simulated cloth mesh using these methods. Texture-based wrinkle modeling method was introduced by Hadap et al. (1999) for cloth simulation [41]. Deformation details will be generated through this method by considering basis as bump map which will be created by a user on a physically simulated bristly cloth object. Kinematic method for generating wrinkles on cloth for Computer Generated (CG) characters was proposed by Cutler et al. (2005) [42]. Through this method, they were able to find out that these similarities could be revealed only on tight fit cloth. Due to that, it was impossible to generate shape detail on loose fit cloth using wrinkle database. A method to generate finely detailed folds on captured cloth model was proposed by Popa et al. (2009) [43]. Shape and position of wrinkles were captured from video footage through this method by considering basis as the distinguishing shape characteristics of wrinkle. Feng et al. (2010) introduced a method to provide high-quality dynamic folds and wrinkle for cloth simulation by maintaining real-time ability [44].Geometrical based virtual clothing method was used to capture the relationship between two different resolutions of mesh and transformation process was executed using this relationship. They developed an animation production pipeline which always starts with physical simulation of low-resolution mesh. Through this method, they have evaluated collisions between each proxy bone instead of calculating collision between each mesh triangles of cloth and body model when a character moves. The computational time that is taken to calculate collisions has reduced considerably even though there is a slight reduction in the accuracy of collision handling. Researchers have been succeeded in improving the efficiency, but a pre-simulation is required to obtain training data and it will be a time-consuming process.

\section{F. Usage of Ease Allowance and Distance Ease Distribution in Pattern Construction}

Ease has become a crucial feature in the clothing industry in order to deliver appropriate garment fit to the wearer. Many researchers have identified a variety of factors that are having a considerable relation to the ease allowance of a garment block pattern construction. Rasband and Liechty (2006) have explained that design style, fabric physical and mechanical attributes, body shape, wearing occasion and personal preference are the main factors that impact the amount of ease needed [45]. Chen, Zeng, Happiette and Bruniaux (2008) have categorized the factors related to ease allowance into three; standard ease, dynamic ease and fabric ease [5]. According to Gill (2011), there are five contributing factors that determine the ease of a garment pattern such as Function, Comfort, Oversize, Fabric and Styling [46]. Huang, Mok, Kwok, \& Au (2012) has identified the amount of clothing ease depends on the design, fabric used, functions of a garment, and even personal preference of customer [4]. Based on these, two types of ease were identified; wearing ease and design ease. As it can be seen, almost all the researchers have considered the shape of the human body and fabric properties to determine ease allowance [4][5][45][46], and these have been identified as having a strong and direct relationship with the distance ease distribution between body and garment [47][48]. Some of the researchers have identified that other than the body shape and fabric properties, the design of the garment, personal preferences and body movements also have a significant impact on the amount of ease allowance required. Still, there are questions left unanswered for the identification of the relationship between these factors and distance ease distribution when a garment is draped upon a human body.

Furthermore, many studies have used 3D body scanning data in order to evaluate the distance ease distribution [4][5][46][47][48][49]. Xu (2008) and Lage (2017) presented the impact on the distance ease between the body and garment by using a variety of fabrics, while uniformly changing the ease allowance. Both studies showed that the 3D distance eases at different body angles changed irregularly with the increase of garment sizes and the uniform changing of material mechanical properties, especially tensile strain [47][48]. Gill (2011) and Wang et al. (2006) have presented different mathematical models of ease distribution which plays an important role in the construction of basic garment patterns [46][50]. Chen et al. (2008) also presented a model to generate optimum ease allowance for the creation and manipulation of pattern construction [5]. Thomassey et al. (2013) identified a template to determine ease in $3 \mathrm{D}$ patterns in order to generate personalized garment patterns [49]. With the identification of distance ease distribution among body and garment, Huang et al. (2012) presented a model to flexibly distribute and accurately control ease in 3D patterns in order to convert those into two-dimensional [4].

Furthermore, studies have been conducted to evaluate the distance ease distribution at the bust section, waist section, abdomen section, hip section, thigh, knee and crotch curve using the respective cross-sections of the body. Moreover, dresses, long-sleeve tops, jackets, trousers have been used for the evaluation of distance ease distribution.

There are still questions left unanswered for determining the garment fit for a given garment type without the usage of 3D technologies as well as for identifying the relationship between the distance-ease distribution and wearer's personal fit preference. 


\section{PROPOSED DESIGN}

The primary focus of this study is to generate a fast online solution which will support the decision-making process of human users, utilizing a less computational power and a less user intervention. The shopper is only required to provide the key body measurements of him or herself along with the selected garment item. These input body measurements of a particular user are the foundation of the human body model generation process is discussed in this part.

The generated body model is then compared with a selected garment item which is available in the online shopping store. As a prerequisite of this proposed solution, the 2D models of the garment items of the online store is required to be generated beforehand and stored in a database.

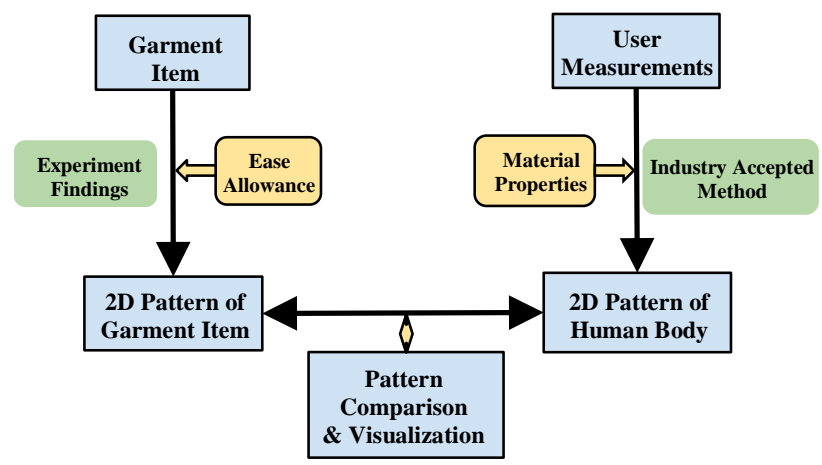

Fig.1. Proposed Design.

\section{A. Model Generation}

The human body and the garment item were modeled in the same platform in order to carry out the comparison process more accurately. In order to represent the models of both human body and garment, a two-dimensional plane was selected as the platform. The concept behind this model generation is representing three-dimensional objects using two-dimensional models preserving its original measurements. In this study two types of models were generated:

- 2D Block Pattern of Garment Item

- 2D Block Pattern of Human Body

\section{- 2 D Block Pattern of Garment Item}

The study focused on representing the garment items using its original 2D block pattern since each garment item is originated by a $2 \mathrm{D}$ block pattern. These block patterns contain the correct measurements that are used to create garments. It is important to identify these correct garment pattern measurements since these are necessary for the comparison process. In the first stage of the study a particular garment item was selected, and it was transformed into its block pattern upon identifying the relationship between the measurements of the finished garment and its block pattern.

An experiment was designed to examine the feasibility of the aforementioned process of transforming a specific clothing item into its block pattern. A male short-sleeve shirt was selected as the garment item for the first attempt of the experiment since it has been a frequent tailor making garment item. The experiment was also aimed at analyzing the relationship between the measurements of the finished garment and its block pattern. At the end of this experiment, the 2D pattern of a finished short-sleeve men's shirt was obtained and plotted using a 2D Cartesian plane. For the experiment ten short-sleeve men's shirts of the same size, same design and same material were selected. The following 15 measurements from the front, back, and sleeve of the shirt were extracted using a measuring tape by placing the garment on a flat surface avoiding wrinkles.

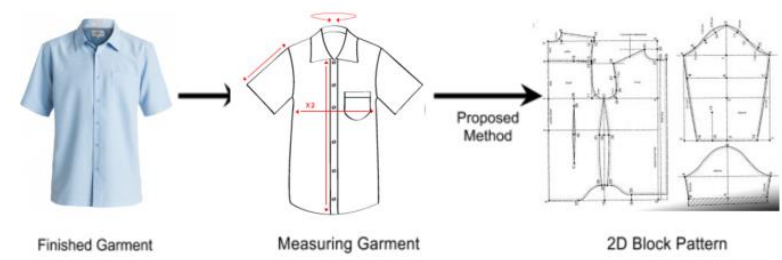

Fig.2. The process of transforming a short-sleeve shirt into its block pattern.

Table 1. Measurements that were taken from the short-sleeve men's shirts

\begin{tabular}{|c|c|c|}
\hline Front & Back & Sleeve \\
\hline $\begin{array}{c}\text { Length from } \\
\text { Shoulder }\end{array}$ & $\begin{array}{c}\text { Length from } \\
\text { Middle }\end{array}$ & Upper Arm Length \\
\hline $\begin{array}{c}\text { Length from } \\
\text { Middle }\end{array}$ & $\begin{array}{c}\text { Length from } \\
\text { Shoulder }\end{array}$ & Under Arm Length \\
\hline Seam Length & Shoulder Width & Lower Girth *2 \\
\hline $\begin{array}{c}\text { Width from Hip } \\
\text { Level }\end{array}$ & $\begin{array}{c}\text { Shoulder Width } \\
\text { from Middle }\end{array}$ & Upper Girth *2 \\
\hline $\begin{array}{c}\text { Width from Waist } \\
\text { Level }\end{array}$ & & \\
\hline $\begin{array}{c}\text { Width from Chest } \\
\text { Level }\end{array}$ & & \\
\hline Collar Width & & \\
\hline
\end{tabular}

After extracting all the necessary garment measurements, the sample shirts were converted into their pre-sewing stage by removing the stitches of the seams; the line where two garment pieces are sewn together. The block patterns of these unstitched garment pieces were drawn on a paper using a tracing wheel which is commonly used in flat pattern making. After that, the previously mentioned 15 measurements were again extracted from the sketches of the block patterns. The differences between the measurements of the finished garment and measurements of the block pattern were analyzed to identify the relationship between the measurements.

The difference between a particular measurement of the garment and the relevant measurement of the block pattern was calculated for each shirt and was compared. It was clearly seen that there were fewer variations among the difference values for each measurement. The problem in this finding was that these results would not be suitable for the generalization of this process for different sizes of short sleeve men's shirts. As it is mentioned before this 
experiment was constrained for the short-sleeve men's regular-fit shirts with the collar size of 15.5 inches. In order to obtain a set of generalized values for all the available sizes of short-sleeve men's shirts, as the second step of the experiment, four different short-sleeve men's shirts with the collar sizes of 15, 15.5, 16 and 16.5 inches were selected and same set of actions of the previous experiment were taken on these four shirts. At the end of this experiment, two graphs were obtained.

\section{Findings of the Experiment}

Conducting the experiment, the relationship of converting the finished garment item (Men's short sleeve shirt) to its 2D block pattern has been identified with following equations for front, back, and the sleeve of the shirt separately.

\section{Half - Front block of the shirt}

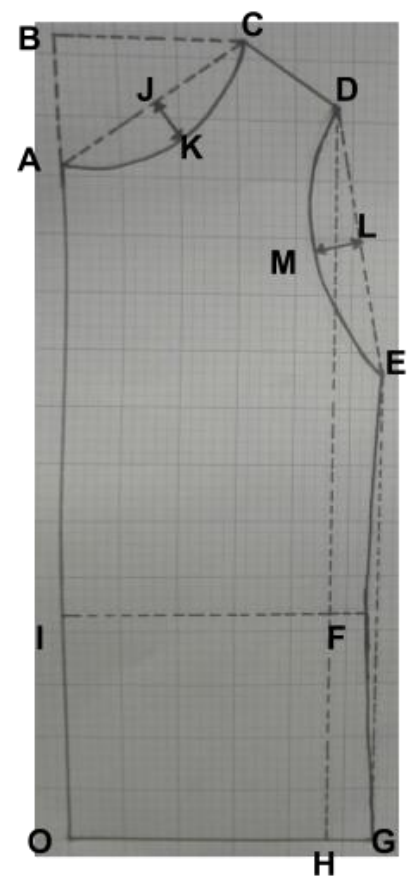

Fig.3. Half front block of the shirt

Table 2. Measurements Taken from Half Front Block of the Shirt

\begin{tabular}{|c|c|}
\hline $\mathrm{OA}$ & Length from the middle of the garment $+b$ \\
\hline $\mathrm{AB}$ & $\begin{array}{l}\text { ( Back length from the middle - Front length } \\
\text { from the middle) }\end{array}$ \\
\hline $\mathrm{BC}$ & Collar width of the garment / 2 \\
\hline $\mathrm{CD}$ & Length from collar to shoulder of the garment \\
\hline $\mathrm{DH}$ & Length from shoulder to hip of the garment $+\mathrm{e}$ \\
\hline $\mathrm{OI}$ & $($ Seam length $+d) / 2$ \\
\hline OG & $($ Lower width of the garment $+a) / 2$ \\
\hline IF & (Waist of the garment $+c$ ) $/ 2$ \\
\hline $\mathrm{AC}$ & $\left(\mathrm{AB}^{2}+\mathrm{BC}^{2}\right)^{1 / 2}$ \\
\hline $\mathrm{JK}$ & $3.5 \mathrm{~cm}$ \\
\hline ML & $4.5 \mathrm{~cm}$ \\
\hline
\end{tabular}

ACK and DME curves can be calculated using the equation; $\mathrm{y}=a \mathrm{x}^{2}+\mathrm{bx}+\mathrm{c}$
JK and ML values are constants and a, b, c, d, e are the values of standard mean errors between the front block and the front of the shirt.

$\mathrm{a}=0.4 \mathrm{~cm} \quad \mathrm{~b}=0.9 \mathrm{~cm} \quad \mathrm{c}=-0.8 \mathrm{~cm} \quad \mathrm{~d}=1.0 \mathrm{~cm} \quad \mathrm{e}=0.9 \mathrm{~cm}$

Half - Back block of the shirt

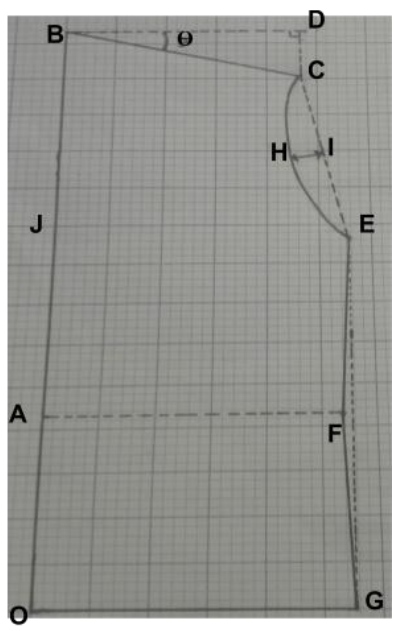

Fig.4. Half back block of the shirt

$$
\begin{gathered}
\cos \Theta=B D / B C \\
\Theta=\cos ^{-1} B D / B C \\
\tan \Theta=n / B D \\
n=\tan \theta(B D)
\end{gathered}
$$

CHE curve can be calculated using the equation;

$$
y=a x^{2}+b x+c
$$

HI value is a constant one and a, b, c, d, e, j, k are the values of standard mean errors between the back block and the back of the shirt

$$
a=0.4 \quad b=1.5 \quad c=1.4 \quad d=1 \quad e=-0.8 \quad j=0.6 \quad k=1.5
$$

Table 3. Measurements Taken from Half Back Block of the Shirt

\begin{tabular}{|c|c|}
\hline OA & (Seam length of the garment $+\mathrm{d}$ ) $/ 2$ \\
\hline $\mathrm{OB}$ & Back length of the middle of the garment $+\mathrm{b}$ \\
\hline $\mathrm{BC}$ & Mid neck to shoulder of the garment $+\mathrm{k}$ \\
\hline $\mathrm{BD}$ & (Full shoulder width of the garment $+\mathrm{j}) / 2$ \\
\hline $\mathrm{DC}$ & Seam length of the garment $+\mathrm{d}$ \\
\hline $\mathrm{EG}$ & ( Chest of the garment $+\mathrm{c}) / 2$ \\
\hline $\mathrm{JE}$ & ( Waist of the garment $+\mathrm{e}) / 2$ \\
\hline $\mathrm{AF}$ & ( Lower width of the garment $+\mathrm{a}) / 2$ \\
\hline $\mathrm{OG}$ & $3.5 \mathrm{~cm}$ \\
\hline $\mathrm{HI}$ & \\
\hline
\end{tabular}

Sleeve block of the shirt 


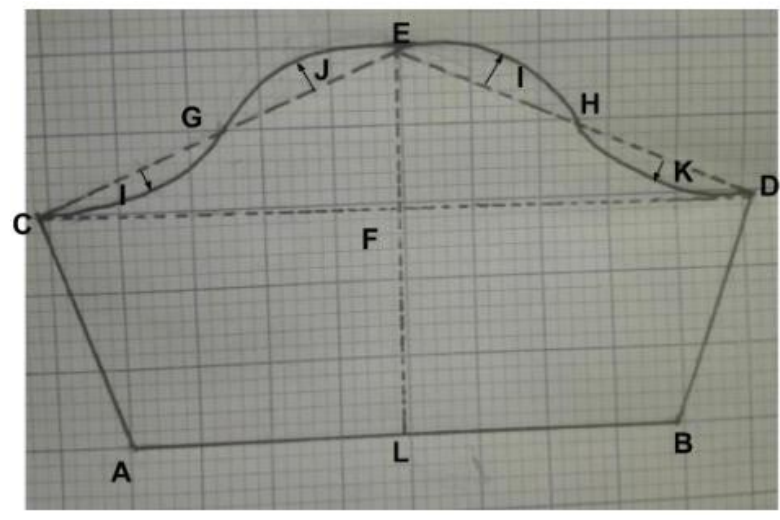

Fig.5. Sleeve block of the shirt

Table 4. Measurements Taken from Sleeve Block of the Shirt

\begin{tabular}{|c|c|}
\hline $\mathrm{AB}$ & Lower arm girth of the garment $+\mathrm{x}$ \\
\hline $\mathrm{CF}$ & (Upper arm girth of the garment $+\mathrm{x}$ ) $/ 2$ \\
\hline $\mathrm{LE}$ & Upper arm length of the garment $+\mathrm{y}$ \\
\hline $\mathrm{FL}$ & Under arm length of the garment $+\mathrm{z}$ \\
\hline $\mathrm{I}$ & $1.25 \mathrm{~cm}$ \\
\hline $\mathrm{J}$ & $1.6 \mathrm{~cm}$ \\
\hline $\mathrm{K}$ & $0.9 \mathrm{~cm}$ \\
\hline
\end{tabular}

$\mathrm{I}, \mathrm{J}, \mathrm{K}$ are constant values and $\mathrm{x}, \mathrm{y}, \mathrm{z}$ are standard mean errors of the sleeve block and the sleeve of the shirt

$$
\mathrm{x}=1.7 \quad \mathrm{y}=0.5 \quad \mathrm{z}=0.15
$$

\section{- $2 D$ Block Pattern of Human Body}

In order to compare the human body with the 2D model of the garment item, the model of the human body was transformed into the same $2 \mathrm{D}$ platform. In the Garment Pattern Making Industry the scientific method for creating 'close-fitting basic bodice block' is used to make garments that are tightly fitted to the male upper body; from the base of the neck to waist, excluding arms. Only the upper body of males was modeled as a basic garment pattern with the use of aforementioned 'closefitting basic bodice block' creation method [51] because of its significant similarity with the representation of the male upper body. 15 body measurements of the male upper body were selected in order to generate this 2D bodice block which is the exact replica of the corresponding user's upper body as the measurements are taken with zero ease allowance. Those measurements are as below.

1. Neck girth

2. Neck base width

3. Chest

4. Hip

5. Waist

6. Side Shoulder-to waist

7. Side neck-to-waist

8. Waist-to-hip

9. Underarm-to-waist

10. Arm hole

11. Shoulder width

12. Shoulder width
13. Upper arm length

14. Under arm length

15. Lower Arm girth

Based on these measurements, 2D patterns of the front, back, and arm of the human body were modeled. Following are the generalized 2D block patterns for a male upper body which can be used to represent different male upper bodies.

Half-Front block of the respective human

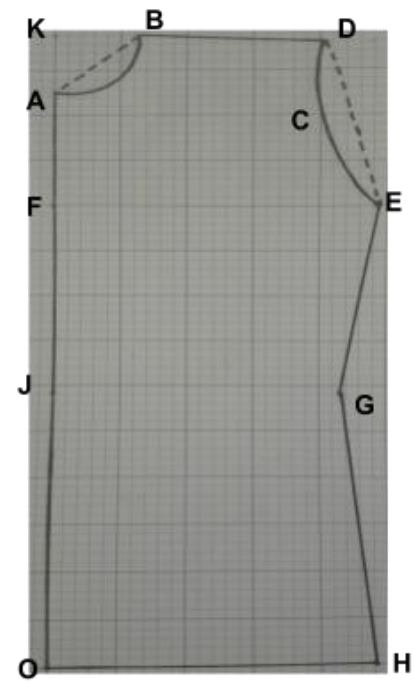

Fig.6. Half front block of the respective human

Table 5. Measurements Taken from Half- Front Block of the Human

\begin{tabular}{|c|c|}
\hline KJ & Side neck to waist \\
\hline DG & Side shoulder to waist \\
\hline JO & Waist-to-hip \\
\hline AK & $($ Neck girth / 6) +0.5 \\
\hline BK & $($ Neck girth / 6) +0.5 \\
\hline KD & Neck base width / \\
\hline FE & $(($ Chest/2)+1) $/ 2$ \\
\hline JG & $(($ Waist $/ 2)+1) / 2$ \\
\hline OH & $(($ Hip/2)+1) / 2 \\
\hline EG & Underarm-to-waist \\
\hline DCE & $($ Armhole / 2) -0.5 \\
\hline
\end{tabular}

Table 6. Measurements Taken from the Half-Back Block of the Human

\begin{tabular}{|c|c|}
\hline DC & Side neck to waist \\
\hline CO & Waist-to-hip \\
\hline AD & 2cm \\
\hline DE & $($ Neck girth / 6) +0.5 \\
\hline DF & Neck base width $/ 2$ \\
\hline BG & $(($ Chest $/ 2)-1) / 2$ \\
\hline CI & $(($ Waist/2)-1) / 2 \\
\hline OJ & $(($ Hip/2)-1) $/ 2$ \\
\hline GI & Underarm-to-waist \\
\hline DCE & $($ Armhole / 2) - 0.5 \\
\hline
\end{tabular}

Half-Back block of the respective human 


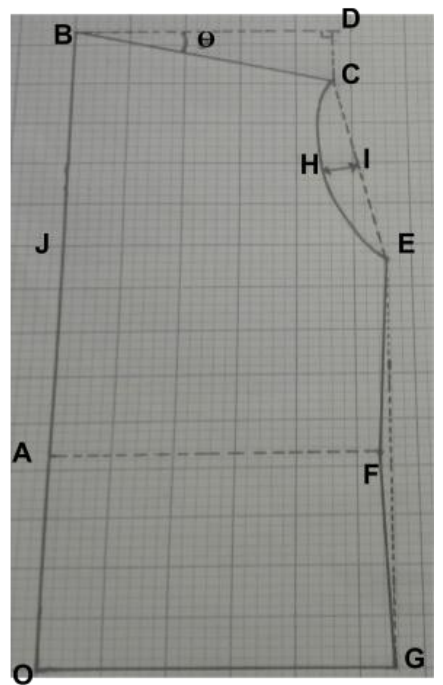

Fig.7. Half back block of the respective human

\section{Arm block of the respective human}

The male arm was modeled as a basic garment pattern with the use of the 'close-fitting sleeve block' creation method. Four measurements of the male arm including upper arm length, under arm length, upper arm girth and lower arm girth were chosen in order to generate the aforementioned 2D sleeve block, which can be considered as an exact model for the corresponding user's arm. The following equations were generated based on the 'close-fitting sleeve block' creation method.

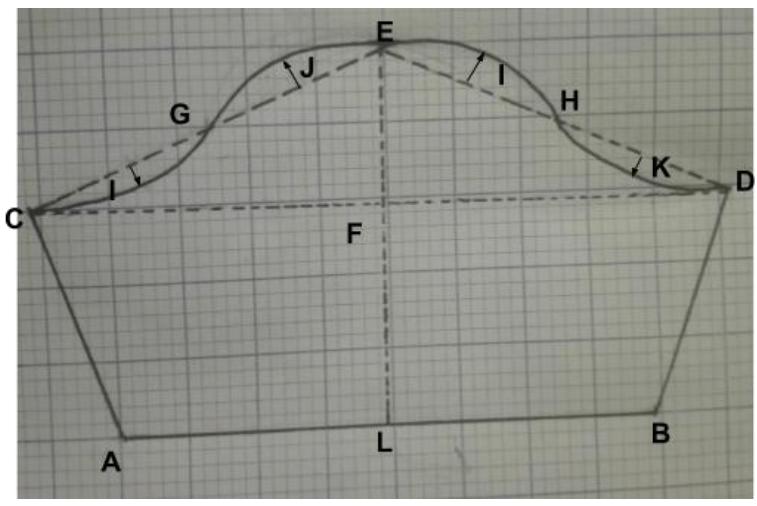

Fig.8. Arm block of the respective human

Table 7. Measurements Taken from the Arm Block of the Human

\begin{tabular}{|c|c|}
\hline EG & Lower arm girth \\
\hline $\mathrm{AD}$ & (Upper arm girth of the garment $+\mathrm{x}$ ) / 2 \\
\hline $\mathrm{DF}$ & Under arm length \\
\hline $\mathrm{BF}$ & Upper arm length \\
\hline $\mathrm{H}$ & $1.25 \mathrm{~cm}$ \\
\hline $\mathrm{I}$ & $1.5 \mathrm{~cm}$ \\
\hline $\mathrm{J}$ & $1.25 \mathrm{~cm}$ \\
\hline $\mathrm{K}$ & $0.9 \mathrm{~cm}$ \\
\hline
\end{tabular}

- Model Comparison and Usage of Distance Ease

The two-dimensional block patterns of male shortsleeve shirt and human body were compared by plotting them on a 2D Cartesian plane as shown in the Fig. 9.

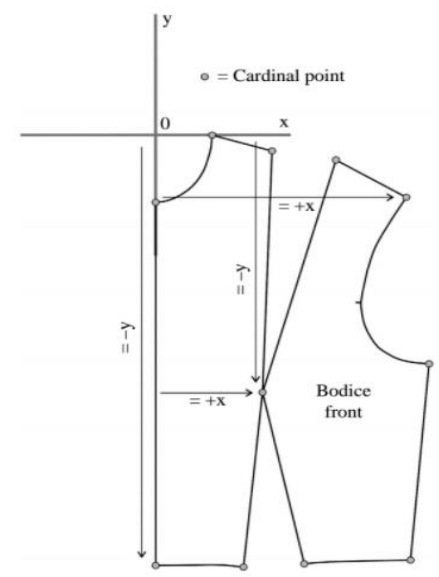

Fig.9. Plotting the block patterns on a 2D Cartesian plane

For the comparison process, the areas of a shirt with most fitting issues were considered. These problematic areas were identified through a survey which was distributed among male university students. As the next step of the comparison process, the relevant numerical points of the two models with respect to the most problematic fitting areas were identified. Upon identifying these numerical points or Cartesian coordinates, the relevant distance values of both models were extracted and compared in order to estimate the fit of the particular garment to that user.

The comparison process was conducted under two main phases. In the first phase, the front block of the garment was compared with the front block pattern of the human upper body. In the second phase, the sleeve block of the garment was compared with the sleeve block pattern of the human upper arm. Moreover, the ease measurements to classify the fitness of the garment has also been proposed in this process based on the literature and ease values for each measurement have been contextualized to the research. The comparison processes conducted under these both phases are furthermore discussed in the following sections.

\section{Model Comparison - Phase I}

When comparing the front block of the garment and the front block pattern of the human upper body, the following areas were considered.

\section{Shoulder}

The shoulder distance (BC) in the shirt with the shoulder distance of human front pattern will be compared. By taking the difference between the shoulder distances of shirt and the human patterns, the user selected shirt can be categorized into the respected fitness level considering the user preference as well. According to the ease values gained through the experiment;

If the difference between shoulder lengths is less than 0.4 , that implies the selected shirt is small for the user. Whether the difference is equal or greater than 0.4 and less than 0.6, the selected shirt can be categorized into the tight or normal fit level. If the difference is equal or 
greater than 0.6 , up to 1.4 the shirt can be categorized into the comfort fitness level and above 1.4, the selected shirt is oversize to the user.

Difference $=($ Shoulder length of the Shirt pattern Shoulder length of the human body pattern)

Small $=$ Difference $<0.4$

Tight fit $=0.6>$ Difference $>=0.4$

Comfort fit $=1.4>$ Difference $>=0.6$

Oversize $=$ Difference $>1.4$

\section{Shirt Length}

The length of the shirt of the front 2D pattern (DH) will be compared with the summation of the side neck to waist (KJ) and waist to hip (JO) lengths in the front human body pattern. Here, length of the human body pattern based on the length expected by the user.

Difference of the Length $=($ Shirt length - Shirt length expected by the user)

Based on the difference get, the output can be displayed as the shirt is $x$ (Difference of the length) shorter/longer than expected length by the user.

\section{Hip}

The hip area of the human body pattern $(\mathrm{OH})$ will be compared with the hip area of the 2D pattern of the shirt (OG). We have calculated the difference between the hip measurement of the shirt and the garment and following comparison criteria were derived based on that difference.

\section{Difference = Shirt hip measurement - Human hip} measurement

Small: Difference $<0.3$

Tight Fit: Difference $<0.8$ and Difference $>=0.3$

Normal Fit: Difference $<1.5$ and Difference $>=0.8$

Comfort Fit: Difference $<2.3$ and Difference $>=1.5$

Oversized garment: Difference $>=2.3$

\section{Chest}

Comparison of chest is done in two phases. In the first phase, chest measurement of human (FE) and chest measurement of shirt will be compared directly. According to our comparison process, if human chest measurement is greater than shirt chest measurement that implies the respective shirt selected by the user is small from the chest area. If the human chest measurement is smaller than or equal shirt chest measurement, it implies that the respective shirt selected by the user needs to analyze further in order to measure the fitness from the chest area. It will be discussed as the second phase of the chest comparison and straight length measurements from side neck to chest level of both shirt and human are needed to proceed this phase. If straight length measurement from side neck to chest level of human is greater than or equal to straight length measurement from side neck to chest level of shirt, it implies that the respective shirt selected by the user is small from the chest area. If straight length measurement from side neck to chest level of human is less than straight length measurement from side neck to chest level of shirt, it implies that the respective shirt selected by the user has no fitness issue from the chest area. Following equations are based on the aforementioned comparison process.

Step 01:

$I F$, chest (human) $>$ chest $($ shirt $)=>$ Shirt is small

IF, chest (human) $<=$ chest $($ shirt $)=>$ Step 02

Step 02:

hO = straight length from side neck to chest level

IF, hO (human) < hO (shirt) $=>$ Shirt is okay

IF, hO (human) $>=h O($ shirt $)=>$ Shirt is small

The fitness of the garment has been classified based on the distance ease preferences as below. We have calculated a proportion in order to determine the fitting criteria and following boundaries were decided based on this proportion.

Proportion $=($ Human chest measurement - Straight length measurement from side neck to chest level of shirt) / (Human waist measurement - Straight length measurement from side neck to chest level of shirt)

Tight Fit: Proportion < 0.6

Normal Fit: Proportion $<1.5$ and Proportion $>=0.6$

Comfort Fit: Proportion $<2.3$ and Proportion $>=1.5$

Oversized Garment: Proportion > $=2.3$

\section{Model Comparison - Phase II}

When comparing the sleeve block of the garment and the sleeve block pattern of the human upper arm the following areas were considered.

\section{Upper Arm girth}

In here the upper arm girth measurement (CD) of the shirt's sleeve block will be compared with the upper arm girth measurement (AC) of the human arm's block pattern. Whenever the $\mathrm{AC}$ measurement $(\mathrm{cm})$ is greater than or equal to the $C D$ measurement $(\mathrm{cm})$ the selected shirt will be considered to be smaller at the arm area for the particular user. If the $\mathrm{AC}$ measurement $(\mathrm{cm})$ is less than the $C D$ measurement $(\mathrm{cm})$ the selected shirt will be considered to be suitable at the arm area for the user. Here the suitability can be again categorized according to the fitness levels.

Difference $=($ Arm girth of the shirt - Arm girth of the human)

Small $=$ Difference $<0.3$

Tight fit $=0.6>$ Difference $>=0.3$

Normal fit $=1>$ Difference $>=0.6$

Comfort fit $=1.5>$ Difference $>=1$

Oversize $=$ Difference $>1.5$

\section{Upper Arm Length}

Here the upper arm length (EL) of the shirt's sleeve 
block will be compared with the upper arm length (BF) of the human arm's block pattern. Whenever the BF measurement $(\mathrm{cm})$ is greater than the EL measurement $(\mathrm{cm})$ the selected shirt sleeve will be considered to be shorter than the user preference of the particular user.

\author{
Arm length (human) > Arm length (shirt) =>Shirt is \\ small \\ Arm length (human) $<=$ Arm length (shirt) =>Shirt is \\ okay
}

\section{CONCLUSION}

There are several methods and tools currently available to check the fitness of a garment with the human body. Most of these techniques demand high computational power since they deliver results utilizing available 3D technologies. Therefore time required for these applications to deliver results is relatively high, and high user intervention is needed to deliver the solution. Further users will access online marketplaces concurrently and it requires high-speed delivery of results in order to control the network traffic. Thus the applicability of aforementioned 3D applications to an online marketplace would be comparatively low. Therefore there is a need for a lightweight solution which will support the decision making of the end user in order to select the properly fitted dress by considering human body properties and garment properties.

\section{REFERENCES}

[1] D. Kim and K. LaBat, "Consumer experience in using 3D virtual garment simulation technology", Journal of the Textile Institute, vol. 104, no. 8, pp. 819-829, 2013.

[2] S. Pheasant and C. Haslegrave, Bodyspace: Anthropometry, Ergonomics and the Design of Work, 3rd ed. Boca Raton, FL.: Taylor \& Francis, 2006.

[3] N. Schofield and K. LaBat, "Exploring the Relationships of Grading, Sizing, and Anthropometric Data", Clothing and Textiles Research Journal, vol. 23, no. 1, pp. 13-27, 2005.

[4] H. Huang, P. Mok, Y. Kwok and J. Au, "Block pattern generation: From parameterizing human bodies to fit feature-aligned and flattenable 3D garments", Computers in Industry, vol. 63, no. 7, pp. 680-691, 2012.

[5] Y. Chen, X. Zeng, M. Happiette, P. Bruniaux, R. Ng and W. Yu, "A new method of ease allowance generation for personalization of garment design", International Journal of Clothing Science and Technology, vol. 20, no. 3, pp. 161-173, 2008.

[6] Cordier, Frédéric et al. "Virtual-Try-On On The Web". VRIC, Virtual Reality International Conference Laval Virtual 2001. Web. 4 May 2017.

[7] D. Protopsaltou, C. Luible, M. Arevalo and N. MagnenatThalmann, "A body and Garment Creation Method for an Internet Based Virtual Fitting Room.", Advances in Modelling, Animation and Rendering, pp. 105-122, 2002.

[8] A. Divivier, Dr. R. Trieb, A. Ebert and H. Hagen, "Virtual Try-On Topics in Realistic, Individualized Dressing in Virtual Reality", Web 4 May 2017.

[9] S. Hauswiesner, M. Straka and G. Reitmayr, "Free viewpoint virtual try-on with commodity depth cameras", Proceedings of the 10th International Conference on Virtual Reality Continuum and Its Applications in Industry - VRCAI '11, 2011.

[10] "OpenNI | http://myrobotlab.org", Myrobotlab.org, 2012. [Online]. Available: http://myrobotlab.org/service/openni. [Accessed: 05- May- 2017].

[11] K. Kortbek, K. Grønbæk and H. Hedegaard, "ARDressCode: Augmented Dressing Room with Tagbased Motion Tracking and Real-Time Clothes Simulation", in Central European Multimedia and Virtual Reality Conference, 2005.

[12] "Follow-the-Trial-Fitter: Real-time dressing without undressing", in Third International Conference on Digital Information Management, London, UK, 2008.

[13] M. Sekine, K. Sugita, F. Perbet, B. Stenger and M. Nishiyama, "Virtual Fitting by Single-shot Body Shape Estimation", in 5th International Conference on 3D Body Scanning Technologies, Lugano, Switzerland, 2014.

[14] F. Cordier, Hyewon Seo and N. Magnenat-Thalmann, "Made-to-measure technologies for an online clothing store", IEEE Computer Graphics and Applications, vol. 23, no. 1, pp. 38-48, 2003.

[15] P. Volino and N. Magnenat-Thalmann, Virtual Clothing, 1st ed. Springer-Verlag GmbH: Springer-Verlag, 2000.

[16] J. Weil, "The synthesis of cloth objects", ACM SIGGRAPH Computer Graphics, vol. 20, no. 4, pp. 49-54, 1986.

[17] Y. Agui and M. Nakajima, "An expression method of cylindrical cloth objects-an expression of folds of a sleeve using computer graphics", 1990.

[18] B. Hinds and J. McCartney, "Interactive garment design", The Visual Computer, vol. 6, no. 2, pp. 53-61, 1990.

[19] B. Hinds, J. McCartney and G. Woods, "Pattern development for 3D surfaces", Computer-Aided Design, vol. 23, no. 8, pp. 583-592, 1991.

[20] C. Calladine, Gaussian curvature and shell structures, The Mathematics of Surfaces, 1st ed. Oxford, England: Clarendon Press, 1986, pp. 179-196.

[21] J. Miller, D. Breen, W. Lorensen, R. O'Bara and M. Wozny, "Geometrically deformed models", ACM SIGGRAPH Computer Graphics, vol. 25, no. 4, pp. $217-$ 226, 1991.

[22] T. Stumpp, J. Spillmann, M. Becker and M. Teschner, "A Geometric Deformation Model for Stable Cloth Simulation", in Workshop on Virtual Reality Interaction and Physical Simulation, 2008.

[23] P. Decaudin, D. Julius, J. Wither, L. Boissieux, A. Sheffer and M. Cani, "Virtual Garments: A Fully Geometric Approach for Clothing Design", Computer Graphics Forum, vol. 25, no. 3, pp. 625-634, 2006.

[24] C. Wang, Y. Wang and M. Yuen, "Feature based 3D garment design through 2D sketches", Computer-Aided Design, vol. 35, no. 7, pp. 659-672, 2003.

[25] T. Igarashi and J. Hughes, "Clothing Manipulation", in 15th Annual ACM Symposium on User Interface Software and Technology, UIST '02, ACM, 2002, pp. 91100.

[26] J. Wang, G. Lu, W. Li, L. Chen and Y. Sakaguti, "Interactive 3D garment design with constrained contour curves and style curves", Computer-Aided Design, vol. 41, no. 9, pp. 614-625, 2009.

[27] Y. Meng, C. Wang and X. Jin, "Flexible shape control for automatic resizing of apparel products", Computer-Aided Design, vol. 44, no. 1, pp. 68-76, 2012.

[28] R. Brouet, A. Sheffer, L. Boissieux and M. Cani, "Design preserving garment transfer", ACM Transactions on 
Graphics, vol. 31, no. 4, pp. 1-11, 2012.

[29] E. Turquin, J. Wither, L. Boissieux, M. Cani and J. Hughes, "A Sketch-Based Interface for Clothing Virtual Characters", IEEE Computer Graphics and Applications, vol. 27, no. 1, pp. 72-81, 2007.

[30] N. Umetani, D. Kaufman, T. Igarashi and E. Grinspun, "Sensitive couture for interactive garment modeling and editing", ACM Transactions on Graphics, vol. 30, no. 4, p. $1,2011$.

[31] D. Terzopoulos, J. Platt, A. Barr and K. Fleischer, "Elastically deformable models", ACM SIGGRAPH Computer Graphics, vol. 21, no. 4, pp. 205-214, 1987.

[32] P. Volino and N. Magnenat-Thalmann, "Accurate Garment Prototyping and Simulation", Computer-Aided Design and Applications, vol. 2, no. 5, pp. 645-654, 2005.

[33] S. Kawabata, M. Niwa and Y. Yamashita, "A guideline for manufacturing "ideal fabrics"", International Journal of Clothing Science and Technology, vol. 11, no. 23, pp. 134-144, 1999.

[34] A. De Boos, D. Tester and A. De Boos, SiroFast, Fabric Assurance by Simple Testing, 1st ed. Geelong: CSIRO Division of Wool Technology, 1997.

[35] P. Volino, N. Magnenat-Thalmann and F. Faure, "A simple approach to nonlinear tensile stiffness for accurate cloth simulation", ACM Transactions on Graphics, vol. 28, no. 4, pp. 1-16, 2009.

[36] M. Chen and K. Tang, "A fully geometric approach for developable cloth deformation simulation", The Visual Computer, vol. 26, no. 6-8, pp. 853-863, 2010.

[37] I. Rudomin, "Simulating cloth using a mixed geometricphysical method", Ph.D, Philadelphia University,USA, 1990.

[38] T. Kunii and H. Gotoda, "Singularity theoretical modeling and animation of garment wrinkle formation processes", The Visual Computer, vol. 6, no. 6, pp. 326-336, 1990.

[39] N. Tsopelas, "Animating the Crumpling Behavior of Garments", in 2nd Eurographics Workshop on Animation and Simulation, 1991, pp. 11-24.

[40] S. Hadap, E. Bangerter, P. Volino and N. MagnenatThalmann, "Animating wrinkles on clothes", in conference on Visualization '99: celebrating ten years, VIS '99, 1999, pp. 175-182.

[41] L. D. Cutler, R. Gershbein, X. Wang, C. Curtis, E. Maigret, L. Prasso and P. Farson, "An art-directed wrinkle system for CG character clothing", in 2005 ACM SIGGRAPH/Eurographics symposium on Computer animation, SCA '05, 2005, pp. 117-125.

[42] T. Popa, Q. Zhou, D. Bradley, V. Kraevoy, H. Fu, A. Sheffer and W. Heidrich, "Wrinkling Captured Garments Using Space-Time Data-Driven Deformation", Computer Graphics Forum, vol. 28, no. 2, pp. 427-435, 2009.

[43] W. Feng, Y. Yu and B. Kim, "A deformation transformer for real-time cloth animation", ACM Transactions on Graphics, vol. 29, no. 4, p. 1, 2010.

[44] J. Rasband and E. Liechty, Fabulous fit, 2nd ed. New York City, NY: Fairchild Publications, 2006.

[45] S. Gill, "Improving garment fit and function through ease quantification", Journal of Fashion Marketing and Management: An International Journal, vol. 15, no. 2, pp. 228-241, 2011.

[46] J. Xu, W. Zhang and P. Xiao, "A study on impact factors of the distance eases between body and garment", in 9th International Conference on Computer-Aided Industrial Design and Conceptual Design, Kunming, China, 2008, pp. 201-205.

[47] A. Lage and K. Ancutiene, "Virtual try-on technologies in the clothing industry. Part 1: investigation of distance ease between body and garment", The Journal of The Textile Institute, pp. 1-7, 2017.

[48] S. Thomassey and P. Bruniaux, "A template of ease allowance for garments based on a 3D reverse methodology", International Journal of Industrial Ergonomics, vol. 43, no. 5, pp. 406-416, 2013.

[49] Z. Wang, E. Newton, R. Ng and W. Zhang, "Ease distribution in relation to the $\mathrm{X}$-line style jacket. Part 1: Development of a mathematical model", Journal of the Textile Institute, vol. 97, no. 3, pp. 247-256, 2006.

[50] W. Aldrich, Metric pattern cutting for menswear. Oxford: BSP Professional Books, 1990.

\section{Authors' Profiles}

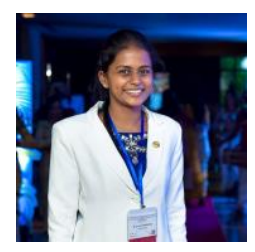

Hashini Gunatilake is a student of University of Colombo School of Computing, Sri Lanka. Currently she is following BSc (Hons) in Information Systems degree and her research interests are basically on Real-time Simulation, ICT based community development, Information Systems, Human-Computer Interaction, eGovernment, IT Strategic Planning for Competitive Advantage and Business Process Management.

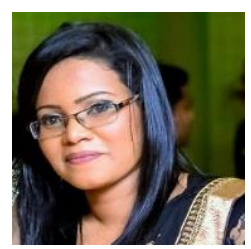

Dulaji Hidellaarachchi is a student of University of Colombo School of Cmputing, Sri Lanka following the BSc (Hons) in Information Systems degree. Her current research interests are Realtime Simulation, ICT based community development, Information Systems, Human-Computer Interaction, e-Government, IT Strategic Planning for Competitive Advantage and Business Process Management.

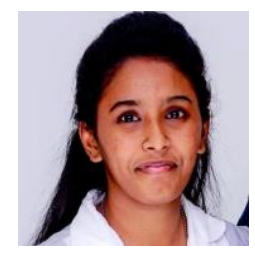

Sandra Perera is a student of University of Colombo School of Computing, Sri Lanka. She is following BSc (Hons) in Information Systems degree and she is currently interested in research areas such as Real-time Simulation, ICT based community development, Information Systems, Human-Computer Interaction, e-Government, IT Strategic Planning for Competitive Advantage and Business Process Management.

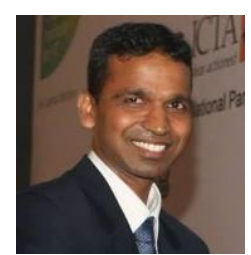

Damitha Sandaruwan received his BSc in Physical Science from the University of Colombo, Sri Lanka, PhD in Computer Science from University of Colombo School of Computing (2015). Media Coordinator of the Advanced Digital Media Technology Centre (ADMTC) UCSC (2008-2014). Co-investigator of the Modelling and Simulation Research Group, UCSC, Sri Lanka. He is currently a Senior Lecturer Gr. II, at the UCSC, Sri Lanka. His Research Interests are Real-time Simulation, Modelling and Simulation, Computer Graphic and Vision. 


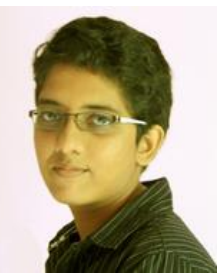

Maheshya Weerasinghe received her BSc in Computer Science (2014) from the University of Colombo School of Computing, Sri Lanka. Student Member of the Computer Society and IEEE Student Branch of University of Colombo School of Computing (2010-2014).

Member of the Modelling and Simulation Research Group, UCSC, Sri Lanka 2014-Present. She is currently a Research Assistant, at the Modelling and Simulation Research Group, UCSC, Sri Lanka. Her Research Interests are Modelling and Simulation, Cognitive Neuroscience, Game based Learning, Cognitive Rehabilitation, and Quantum Computing.

How to cite this paper: Hashini Gunatilake, Dulaji Hidellaarachchi, Sandra Perera, Damitha Sandaruwan, Maheshya Weerasinghe, "An ICT Based Solution for Virtual Garment Fitting for Online Market Place", International Journal of Information Technology and Computer Science(IJITCS), Vol.10, No.2, pp.60-72, 2018. DOI: 10.5815/ijitcs.2018.02.06 